Artículo científico

Volumen 32(1): 149-162. Enero-abril, 2021

e-ISSN 2215-3608, doi:10.15517/am.v32i1.40573

https://www.revistas.ucr.ac.cr/index.php/agromeso

\title{
Sensibilidad de Phytophthora infestans a extractos acuosos de Lippia origanoides y Origanum vulgare ${ }^{l}$
}

\section{Sensitivity of Phytophthora infestans to aqueous extracts of Lippia origanoides and Origanum vulgare}

\author{
Diana Burbano-David ${ }^{2}$,Luz Estela Lagos-Mora ${ }^{3}$, Sandra Álvarez-Ordoñez ${ }^{3}$, Harold Andrés Chañag-Miramag ${ }^{3}$
}

1 Recepción: 5 de febrero, 2020. Aceptación: 16 de setiembre, 2020. Este trabajo se desarrolló dentro del marco del Programa "Fortalecimiento de capacidades regionales en investigación, desarrollo tecnológico e innovación en el departamento de Nariño" en su componente de "Jóvenes Investigadores e Innovadores" financiado por la Gobernación de Nariño, Colombia.

2 Corporación Colombiana de Investigación Agropecuaria (AGROSAVIA), Profesional de Apoyo a la Investigación, Colombia. dmburbano@ agrosavia.co (autora para la correspondencia, http://www.orcid.org/0000-0003-0109-3088).

3 Universidad de Nariño, Grupo Genética y Evolución de Organismos Tropicales (GENPAT). Ciudadela Universitaria Torobajo, Pasto, Colombia. luzestelal@gmail.com (https://orcid.org/0000-0002-5152-3413); diglossandra@gmail.com (https://orcid.org/0000-0002-9464-5429); harold.a963@hotmail.com (https://orcid.org/0000-0002-2749-0367).

\section{Resumen}

Introducción. Phytophthora infestans (Mont.) de Bary, es considerado el agente causal de una de las enfermedades más devastadoras y limitantes de cultivos de papa (Solanum tuberosum L.) en el departamento de Nariño (Colombia). Esta enfermedad es controlada con fungicidas sistémicos, los cuales tienen un impacto negativo sobre el ambiente e incrementan los costos de producción, sin embargo, existen alternativas como los denominados fungicidas orgánicos, los cuales se basan en el uso de extractos de plantas con propiedades antifúngicas para el control del patógeno. Objetivo. Evaluar extractos acuosos de $O$. vulgare y L. origanoides para el control de $P$. infestans bajo condiciones in vitro. Materiales y métodos. La presente investigación se desarrolló en el Laboratorio de microbiología del grupo de investigación en Genética y Evolución de Organismos Tropicales (GENPAT) de la Universidad de Nariño, durante el año 2017. Se evaluó la sensibilidad in vitro de P. infestans frente a extractos de las especies botánicas Lippia origanoides (orégano silvestre) y Origanum vulgare (orégano común). Resultados. Los datos obtenidos permitieron el establecimiento de la línea base de comportamiento de las poblaciones del patógeno frente a los compuestos evaluados, hubo una reducción gradual tanto en el crecimiento como en la esporulación cuando se empleó el extracto de Lippia origanoides y que este mismo tiene la capacidad de controlar el patógeno en concentraciones de $100 \mathrm{mg} \mathrm{ml}^{-1}$. Con el empleo de Origanum vulgare los aislamientos de $P$. infestans presentaron diferentes niveles de sensibilidad al compuesto con bajos niveles de inhibición y altos valores de EC-50. Conclusión. Los aislamientos de $P$. infestans presentaron diferentes niveles de sensibilidad frente a los dos extractos acuosos evaluados, con una reducción del $100 \%$ en la inhibición del crecimiento radial y la esporulación a partir de la concentración de $100 \mathrm{mg} \mathrm{ml}^{-1}$, cuando se empleó el extracto de L. origanoides.

Palabras clave: extractos vegetales; tizón tardío, orégano, Solanum tuberosum, fungicidas orgánicos. 


\begin{abstract}
Introduction. Phytophthora infestans (Mont.) De Bary, is considered the causative agent of one of the most devastating and limiting diseases of potato crops (Solanum tuberosum L.) in the Nariño department (Colombia). This disease is controlled using systemic fungicides, which have a negative impact on the environment and increase production costs, however, there are alternatives like the organic fungicides, which are based on the use of plant extracts with anti-fungal properties for the control of the pathogen. Objective. To evaluate aqueous extracts of $O$. vulgare and L. origanoides for the control of $P$. infestans under in vitro conditions. Materials and methods. This research was carried out in the Microbiology laboratory of the research group in Genetics and Evolution of Tropical Organisms (GENPAT) of the Universidad de Nariño, during 2017. The in vitro sensitivity of $P$. infestans against extracts of botanical species Lippia origanoides (wild oregano) and Origanum vulgare (common oregano) was evaluated. Results. The data obtained allowed the establishment of the base line of behavior of the pathogen populations against the evaluated compounds, there was a gradual reduction in both the growth and sporulation when the Lippia origanoides extract was used and that this same has the capacity to control the pathogen in concentrations of $100 \mathrm{mg} \mathrm{ml}^{-1}$. With the use of Origanum vulgare the P. infestans isolates showed different levels of sensitivity to the compound with low levels of inhibition and high values of EC-50. Conclusion. The $P$. infestans isolates presented different levels of sensitivity against the two aqueous extracts evaluated, with a reduction of $100 \%$ in the inhibition radial growth and sporulation from the concentration of $100 \mathrm{mg} \mathrm{ml}^{-1}$, when the extract was used of $L$. origanoides.
\end{abstract}

Keywords: plant extracts, late blight, oregano, Solanum tuberosum, organic fungicides.

\title{
Introducción
}

La gota o tizón tardío es una de las enfermedades más devastadoras de cultivos de papa (Solanum tuberosum L.), su agente causal, el oomycete Phytophthora infestans (Mont.) de Bary posee una alta plasticidad genética y altos niveles de virulencia (Silva et al., 2009). La presencia del patógeno en este cultivo ha causado pérdidas económicas de hasta el $100 \%$ y su incidencia se atribuye a la siembra de material susceptible, la disponibilidad de fuentes constantes de inóculo en campo y la ubicación de sus hospederos en regiones ambientalmente favorables para el establecimiento del patógeno (Garrett et al., 2001; García et al., 2008).

En Colombia y, especialmente en el departamento de Nariño, uno de los cultivos que más aportan al sector económico es la papa, debido a que es el segundo cultivo con mayor área sembrada (19 046 ha), con una producción de 398 851,47 t para el año 2018 (Red de Información y Comunicación del Sector Agropecuario de Colombia, 2018) y uno de los productos más importantes de las zonas altas de la región. Sin embargo, pese a la importancia del tubérculo en el sector productivo, económico y social, además de su alto potencial de comercialización, los agricultores se enfrentan a problemas fitosanitarios que se traducen en bajos rendimientos de las cosechas con pérdidas anuales de hasta el $40 \%$ (Santa et al., 2018) y altos costos de producción, debido a que no se cuenta con un plan de manejo integrado y sostenible para el control del patógeno.

Las medidas de prevención y control que se toman en los cultivos de papa frente a la gota o tizón tardío, se han orientado a la destrucción de fuentes iniciales del inóculo, la siembra de semilla certificada y la aplicación de fungicidas (Rivera et al., 2010); sin embargo, el manejo de plagas y enfermedades requiere una entrada extremadamente alta de pesticidas (Santa et al., 2018), que generan problemas medioambientales y provocan el desarrollo de nuevos genotipos del patógeno con sensibilidad reducida a las moléculas, lo que más difícil su control.

De acuerdo con esta problemática y con perspectivas hacia un manejo sostenible del cultivo, es necesario plantear alternativas para optimizar el manejo integrado de la enfermedad. Con este fin, se han investigado 
diferentes sustancias de origen biótico y abiótico como posibles soluciones amigables con el ambiente que permitan el manejo de enfermedades. Una de las estrategias que se ha utilizado en torno a esta temática es el manejo sostenible de plagas y enfermedades, donde se busca la implementación de un conjunto de prácticas integrales entre las que se incluye el uso de biopreparados, los cuales se clasifican teniendo en cuenta su forma de acción como bioestimulantes, biofertilizantes, bionsecticidas o biofungicidas (International Panel of Experts on Sustainable Food Systems / Food and Agriculture Organization, 2010).

Bajo este contexto, el uso de extractos vegetales y, especialmente, de algunos de sus compuestos orgánicos, puede ser un método muy atractivo para el control de enfermedades. Es así como algunas investigaciones han centrado sus esfuerzos en el estudio de las propiedades antifúngicas de extractos de especies botánicas, los cuales han generado gran interés por sus resultados a nivel in vitro e in vivo (Dakole et al., 2016; Mohsan et al., 2017; Nagar et al., 2017; Debjani et al., 2018) para el desarrollo de los llamados fungicidas orgánicos o biofungicidas. En los casos particulares de Lippia origanoides y Origanum vulgare, se han reportado la presencia de compuestos fenólicos naturales, entre los que se encuentran timol y carvacrol, considerados como posibles agentes antifúngicos y antibacterianos (Arámbula et al., 2019). Este tipo de estrategias permiten al agricultor un mejor aprovechamiento de las alternativas agroecológicas y menores requerimientos de control con insumos químicos.

Los mecanismos de acción de los monoterpenos timol y carvacrol aún no han sido completamente dilucidados; sin embargo, se conoce que la presencia y la posición de un grupo hidroxilo en la estructura del compuesto puede estar relacionada con su actividad antifúngica (Ultee et al., 2002; Numpaque et al., 2011; Wang et al., 2019). Adicionalmente, se conoce que dichos grupos hidroxilo son indispensables en el establecimiento de enlaces de hidrógeno y en la liberación de protones (Ultee et al., 2002). Se ha reportado que pueden estar involucrados en la ruptura de la membrana por compuestos lipofílicos, afectar la homeostasis del $\mathrm{pH}$ y gradientes de $\mathrm{K}^{+}$, lo que da como resultado un desequilibrio hídrico, reducción de ATP intracelular y muerte celular (Ahmad et al., 2011; Arámbula et al., 2019).

Debido a que las especies botánicas Lippia origanoides y Origanum vulgare presentan actividad antimicrobiana asociada con la presencia de compuestos aromáticos y grupos fenólicos (García-Camarillo et al., 2006; BetancurGalvis et al., 2011), además del aprovechamiento de especies vegetales que se encuentran en estado silvestre como es el caso del L. origanoides; se planteó como objetivo de esta investigación evaluar extractos acuosos de $O$. vulgare y L. origanoides para el control de P. infestans bajo condiciones in vitro.

\section{Materiales y métodos}

\section{Localización}

El estudio se realizó durante los años 2016 y 2017, en el laboratorio de microbiología del grupo de investigación Genética y Evolución de Organismos Tropicales (GENPAT) de la Universidad de Nariño, ubicado en el municipio de Pasto, Nariño, Colombia, con una altitud de $2527 \mathrm{msnm}$, temperatura promedio de $13,8{ }^{\circ} \mathrm{C}$, precipitación anual de $1273 \mathrm{~mm}$ y humedad relativa promedio del $73 \%$.

\section{Diseño experimental}

Para la evaluación de susceptibilidad se empleó un diseño completamente al azar con cuatro tratamientos, tres réplicas técnicas y tres repeticiones biológicas. Las pruebas in vitro se realizaron con diecinueve aislamientos de P. infestans procedentes de cultivos de Solanum tuberosum y Solanum phureja en las zonas productoras de papa del departamento de Nariño, de las cuales hacen parte del cepario del grupo de investigación GENPAT (Cuadro 1). 
Cuadro 1. Información sobre los aislamientos $P$. infestans utilizados en el estudio de extractos acuosos de O. vulgare y L. origanoides para el control de P. infestans bajo condiciones in vitro. Laboratorio de microbiología del grupo de investigación en Genética y Evolución de Organismos Tropicales (GENPAT) de la Universidad de Nariño, Colombia. 2017.

Table 1. Information about $P$. infestans isolates used in the study of aqueous extracts of $O$. vulgare and L. origanoides for the control of $P$. infestans under in vitro conditions. Microbiology laboratory of the research group in Genetics and Evolution of Tropical Organisms (GENPAT) of the Universidad de Nariño, Colombia. 2017.

\begin{tabular}{|c|c|c|}
\hline Código aislamiento & Localidad & Hospedero \\
\hline SPP031 & Pasto & S.phureja \\
\hline SPT056 & Túquerres & S. phureja \\
\hline STA078 & Aldana & S. tuberosum \\
\hline STA083 & Aldana & S. tuberosum \\
\hline STA084 & Aldana & S. tuberosum \\
\hline STA 157 & Aldana & S. tuberosum \\
\hline STG075 & Guachucal & S. tuberosum \\
\hline STG100 & Guachucal & S. tuberosum \\
\hline STI003 & Ipiales & S. tuberosum \\
\hline STI006 & Ipiales & S. tuberosum \\
\hline STI155 & Ipiales & S. tuberosum \\
\hline STI168 & Ipiales & S.tuberosum \\
\hline STP039 & Pasto & S. tuberosum \\
\hline STP041 & Pasto & S.tuberosum \\
\hline STP046 & Río Bobo & S. tuberosum \\
\hline STP062 & Pasto & S. tuberosum \\
\hline STP067 & Pasto & S.tuberosum \\
\hline STP115 & Pasto & S. phureja \\
\hline STT162 & Túquerres & S. tuberosum \\
\hline
\end{tabular}

\section{Obtención del extracto vegetal}

El material vegetal de $O$. vulgare se obtuvo de huertas caseras ubicadas en el municipio de Pasto. En el caso de L. origanoides, la colecta se realizó en un área subxerofítica en Alto Patía, Nariño, donde las plantas se encontraban en condiciones silvestres. Una vez colectadas las hojas, se transportaron al laboratorio donde se procedió a realizar el secado del material a temperatura ambiente durante diez días.

Para la obtención de los extractos acuosos, se pesaron $20 \mathrm{~g}$ de material vegetal y se dispusieron en un Erlenmeyer de $500 \mathrm{ml}$ que contenían $200 \mathrm{ml}$ de agua destilada, los matraces fueron sellados con tapones de algodón y esterilizados en autoclave durante $45 \mathrm{~min}$ a $121{ }^{\circ} \mathrm{C}$ (1 atm). Posteriormente, el líquido fue filtrado empleando tres capas de gasa estéril, el volumen obtenido se llevó a ebullición hasta obtener un volumen aproximado de $20 \mathrm{ml}$. Los extractos se centrifugaron para eliminar los residuos y se esterilizaron nuevamente en autoclave durante 20 min a $121{ }^{\circ} \mathrm{C}$ (1atm); finalmente, los extractos se almacenaron a $4{ }^{\circ} \mathrm{C}$ (Widmer \& Laurent, 2006).

\section{Sensibilidad in vitro de $P$. infestans a extractos vegetales}

Los dos extractos vegetales se evaluaron con base en la metodología del medio de cultivo envenenado (Ochoa-Fuentes et al., 2012) y de diferentes concentraciones calculadas a partir de una escala logarítmica $(0,1$, 
1,10 y $100 \mathrm{mg} \mathrm{ml}^{-1}$ ). Para la obtención de las concentraciones, se empleó el bioinsumo a una concentración de 1

$\mathrm{g} \mathrm{ml}^{-1} \mathrm{y}$, posteriormente, se realizaron las diluciones empleando como solvente el medio de cultivo hasta obtener las concentraciones deseadas. Todos los ensayos contaron con un control negativo, el cual consistió en medio de cultivo sin suplementar con extracto vegetal.

Un disco de $5 \mathrm{~mm}$ se extrajo de la zona activa de crecimiento de cultivos de diez días de P. infestans y se ubicó en el centro de cada caja petri que contenía agar tomate-arveja previamente suplementado con el extracto vegetal. Una vez efectuada la inoculación, las cajas petri se sellaron y se mantuvieron a temperatura ambiente y oscuridad durante diez días. Terminado el periodo de incubación, se realizó un registro fotográfico, las imágenes obtenidas se procesaron con el software ImageJ 1,45 s (Waine Rasband, National Institutes of Health, USA) y se calculó el porcentaje de inhibición de crecimiento radial (PICR) aplicando la fórmula PICR $(\%)=(\mathrm{c}-\mathrm{t} / \mathrm{c})$ x 100; donde: c: área de crecimiento micelial en el tratamiento control y t: área de crecimiento micelial en el tratamiento con extracto (You et al., 2016).

Al décimo día se tomó el micelio formado en cada caja petri y se depositó en un volumen de 1,5 ml de agua destilada estéril, se agitó en vortex por $30 \mathrm{~s}$, se tomaron $10 \mu \mathrm{l}$ de esta solución para realizar el conteo de esporangios en cámara de Neubauer y se expresó como número de esporangios sobre el área de crecimiento final para cada uno de los aislamientos por tratamiento.

Se determinó la dosis media efectiva (EC-50) de los extractos vegetales por cada aislamiento mediante un análisis Probit con los valores de inhibición y concentración obtenidos para cada uno de los aislamientos basados en un análisis de regresión lineal.

\section{Análisis de datos}

Con los resultados obtenidos para porcentaje de inhibición y tasas de esporulación se elaboraron cuadros de contingencia, los datos se sometieron a pruebas de normalidad y de homogeneidad de varianza; comprobados los supuestos, se realizó análisis de varianza de un solo factor (ANOVA) en el caso de los datos que presentaron distribución normal y un análisis de Kruskal-Wallis para los resultados que no mostraban dicha distribución, con el fin de comparar dichos parámetros en las diferentes concentraciones con el programa Minitab® 17.1.0 (ㅇ 2013 Minitab Inc).

\section{Resultados}

\section{Susceptibilidad de $P$. infestans frente a extracto acuoso de Lippia origanoides}

Todas las concentraciones evaluadas redujeron el crecimiento radial de los diferentes aislamientos de $P$. infestans (Figura 1) a partir de la concentración de $0,1 \mathrm{mg} \mathrm{ml}^{-1}$. Se incrementó la inhibición a medida que aumentó el contenido del extracto acuoso en el medio de cultivo (Cuadro 2). En las dos primeras dosis evaluadas (0,1 y 1 $\mathrm{mg} \mathrm{ml}^{-1}$ ), se encontró que los porcentajes de inhibición no superaron el $40 \%$, mientras que en la concentración de $10 \mathrm{mg} \mathrm{ml}^{-1}$ los porcentajes variaron entre el 21 y el $100 \%$, en el caso de la concentración de $100 \mathrm{mg} \mathrm{ml}^{-1}$ todos los aislamientos inhibieron su crecimiento completamente.

A pesar de la eficiencia del extracto vegetal en el control in vitro del patógeno, los resultados mostraron que en total el $37 \%$ de los aislamientos tuvieron un crecimiento radial mayor al presentado en el tratamiento control, seis de los aislamientos presentaron este comportamiento en la concentración $0,1 \mathrm{mg} \mathrm{ml}^{-1}$, y un aislamiento en la concentración de $1 \mathrm{mg} \mathrm{ml}^{-1}$. El análisis de varianza mostró que existen diferencias estadísticamente significativas $(p=0,000, g l=75, \alpha=0,05)$, dichas diferencias se presentaron entre todas las concentraciones evaluadas. 


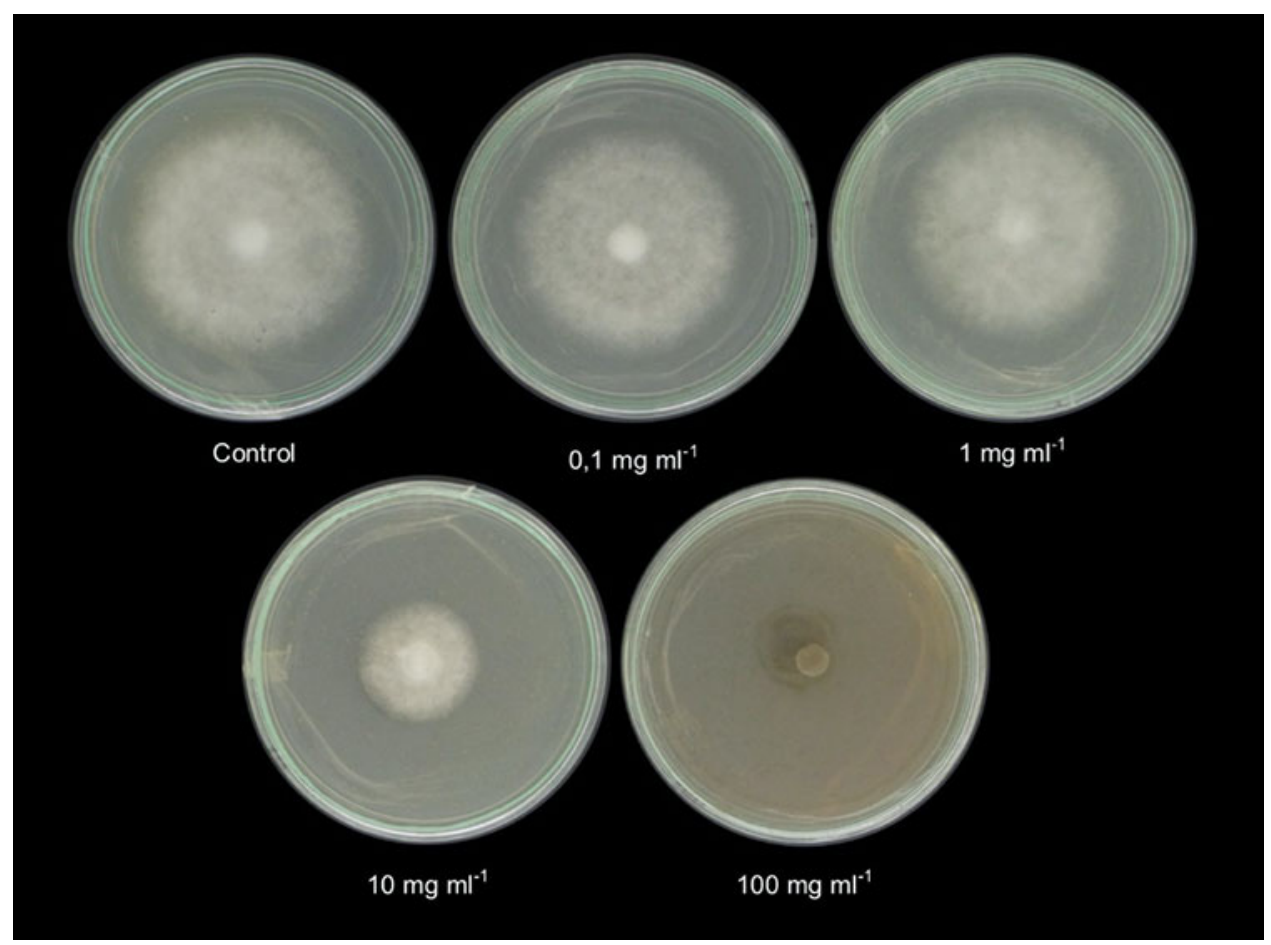

Figura 1. Sensibilidad de P. infestans (aislamiento STG100) a diferentes concentraciones del extracto acuoso de L. origanoides. Laboratorio de microbiología del grupo de investigación en Genética y Evolución de Organismos Tropicales (GENPAT) de la Universidad de Nariño, Colombia. 2017.

Figure 1. Sensitivity of Phytophthora infestans (STG100 isolate) to different concentrations of the aqueous extract of L. origanoides. Microbiology laboratory of the research group in Genetics and Evolution of Tropical Organisms (GENPAT) of the Universidad de Nariño, Colombia. 2017.

Respecto a la tasa de esporulación se encontró que la reducción del 100 \% en la producción de esporangios solo se presentó en la última concentración evaluada $\left(100 \mathrm{mg} \mathrm{ml}^{-1}\right)$, al igual que los resultados presentados para porcentaje de inhibición. La prueba estadística Kruskal-Wallis mostró diferencias estadísticas $(\mathrm{p} \leq 0,05)$ entre la concentración de $100 \mathrm{mg} \mathrm{ml}^{-1}$ y las demás dosis evaluadas (Cuadro 3). A excepción de la concentración de 100 $\mathrm{mg} \mathrm{ml}^{-1}$, en los demás casos se evidenció una alta producción de esporangios por parte del patógeno, con tasas de esporulación promedio de 13 734, 11 171, 9632 y 12630 esporangios $\mathrm{cm}^{-2}$ para las concentraciones de $0,0,1,1$ y $10 \mathrm{mg} \mathrm{ml}^{-1}$, respectivamente.

A partir de los resultados de inhibición se obtuvo el valor de la dosis media efectiva (EC-50); para el caso del extracto de Lippia origanoides, estos variaron entre 2,55 mg ml${ }^{-1}$ en el aislamiento STI006 y hasta 13,63 mg ml-1 en el aislamiento STP067, con un valor promedio de 5,66 $\mathrm{mg} \mathrm{ml}^{-1}$ (Figura 2).

\section{Susceptibilidad de $P$. infestans frente a extracto acuoso de Origanum vulgare}

En los ensayos realizados con $O$. vulgare se observó que las concentraciones empleadas redujeron el crecimiento radial del patógeno (Figura 3). El $21 \%$ de los aislamientos presentaron crecimiento por encima del tratamiento control, dos de ellos en la concentración $0,1 \mathrm{mg} \mathrm{ml}^{-1}$ y dos aislamientos más presentaron el mismo 
Cuadro 2. Porcentaje de Inhibición de crecimiento radial (PICR), de aislamientos de Phytophthora infestans después de diez días expuesto a extracto acuoso de L. origanoides a diferentes concentraciones. Laboratorio de microbiología del grupo de investigación en Genética y Evolución de Organismos Tropicales (GENPAT) de la Universidad de Nariño, Colombia. 2017.

Table 2. Inhibition percentage on radial growth (IPRG), of Phytophthora infestans isolates after ten days exposed to aqueous extract of L. origanoides at different concentrations. Microbiology laboratory of the research group in Genetics and Evolution of Tropical Organisms (GENPAT) of the Universidad de Nariño, Colombia. 2017.

\begin{tabular}{ccccc}
\hline & \multicolumn{4}{c}{ Concentración $\left.\mathbf{( m g ~ m l}^{-\mathbf{1}}\right)^{*}$} \\
Aislamiento & $\mathbf{0 , 1}$ & $\mathbf{1}$ & $\mathbf{1 0}$ & $\mathbf{1 0 0}$ \\
\hline SPP031 & $0,5 \pm 1,3$ & $14,3 \pm 2,8$ & $82,1 \pm 1,8$ & $100 \pm 0$ \\
SPT056 & $6,2 \pm 0,6$ & $21,3 \pm 1,7$ & $36,6 \pm 0,5$ & $100 \pm 0$ \\
STA078 & $-3,1 \pm 1,2$ & $19,3 \pm 1,2$ & $68,1 \pm 0,5$ & $100 \pm 0$ \\
STA083 & $-23,8 \pm 2,7$ & $5,5 \pm 1,5$ & $70,8 \pm 1,5$ & $100 \pm 0$ \\
STA084 & $-25 \pm 0,8$ & $26,9 \pm 1,6$ & $94,5 \pm 0,5$ & $100 \pm 0$ \\
STA157 & $3,9 \pm 1,9$ & $22,4 \pm 0,9$ & $51,1 \pm 1,2$ & $100 \pm 0$ \\
STG075 & $8,5 \pm 1,7$ & $17,2 \pm 2,2$ & 67,92 & $100 \pm 0$ \\
STG100 & $10,9 \pm 1$ & $15,6 \pm 2,8$ & $67,7 \pm 2$ & $100 \pm 0$ \\
ST1003 & $11,1 \pm 0,6$ & $5,6 \pm 0,6$ & $20,4 \pm 0,4$ & $100 \pm 0$ \\
ST1006 & $-3,8 \pm 1,5$ & $32,2 \pm 0,8$ & $85,3 \pm 1,1$ & $100 \pm 0$ \\
ST155 & $8,6 \pm 1,2$ & $9,3 \pm 0,7$ & $69,2 \pm 1,6$ & $100 \pm 0$ \\
ST1168 & $9,4 \pm 1,7$ & $4,7 \pm 2,1$ & $71 \pm 1,4$ & $100 \pm 0$ \\
STP039 & $3,7 \pm 1,4$ & $9,8 \pm 1,3$ & $46,3 \pm 1,5$ & $100 \pm 0$ \\
STP041 & $18,3 \pm 1,2$ & $7,5 \pm 1,4$ & $67,5 \pm 2,4$ & $100 \pm 0$ \\
STP046 & $12,7 \pm 2,6$ & $-22,1 \pm 1,4$ & $37,5 \pm 1,1$ & $100 \pm 0$ \\
STP062 & $0,4 \pm 2,4$ & $19 \pm 0,8$ & $61,8 \pm 1,6$ & $100 \pm 0$ \\
STP067 & $-12,7 \pm 2,3$ & $3,1 \pm 1,3$ & $19,6 \pm 2,3$ & $100 \pm 0$ \\
STP115 & $-46,1 \pm 2,4$ & $27,6 \pm 2,5$ & $76,8 \pm 1$ & $100 \pm 0$ \\
STT162 & $5,7 \pm 1,3$ & $3,1 \pm 2,4$ & $54,3 \pm 2,7$ & \\
\hline
\end{tabular}

* Los datos corresponde a la media de nueve repeticiones $(\mathrm{n}=9) ; \pm$ corresponde a la desviación estándar / The data corresponds to the mean of nine replications $(\mathrm{n}=9) ; \pm$ corresponds to the standard deviation.

Cuadro 3. Prueba Kruskal-Wallis (Bonferroni corrected) para tasa de esporulación entre concentraciones del extracto acuoso de $L$. origanoides. Laboratorio de Microbiología del Grupo de Investigación en Genética y Evolución de Organismos Tropicales (GENPAT) de la Universidad de Nariño, Colombia. 2017.

Table 3. Kruskal-Wallis test (Bonferroni corrected) for sporulation rate between concentrations of the aqueous extract of $L$. origanoides. Universidad de Nariño, Microbiology Laboratory - Genpat, Colombia. 2017.

\begin{tabular}{cccccc}
\hline Concentración $\left(\mathbf{m g ~ m l}^{-1}\right)$ & Control & $\mathbf{0 , 1}$ & $\mathbf{1}$ & $\mathbf{1 0}$ & $\mathbf{1 0 0}$ \\
\hline Control & 1 & & & & \\
$\mathbf{0 , 1}$ & 1 & 1 & 1 & & \\
$\mathbf{1}$ & 1 & 1 & 1 & 1 & 1 \\
$\mathbf{1 0}$ & 1 & 1 & 0,02621 & \\
$\mathbf{1 0 0}$ & 0,0001942 & 0,0001942 & 0,0007247 & 0,0267 & \\
\hline
\end{tabular}




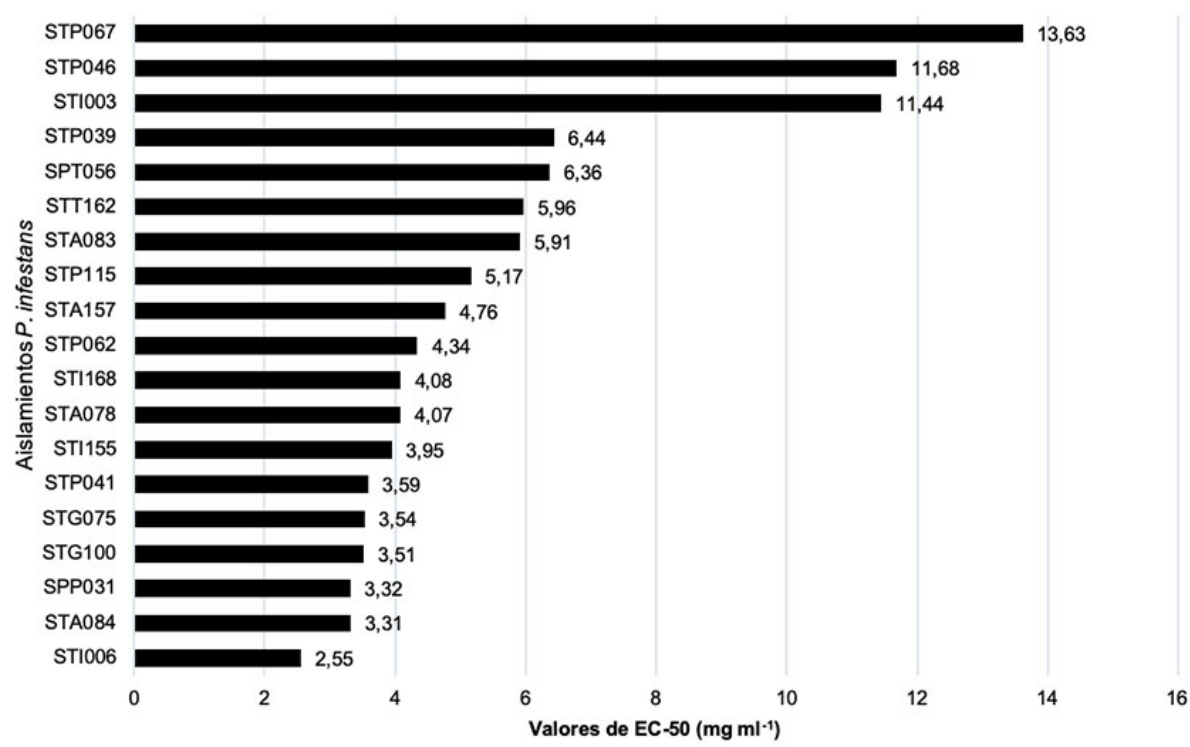

Figura 2. Valor dosis media efectiva (EC-50) encontrados al evaluar la sensibilidad de diferentes concentraciones de extracto vegetal de L. origanoides en aislamientos de P. infestans. Laboratorio de microbiología del grupo de investigación en Genética y Evolución de Organismos Tropicales (GENPAT) de la Universidad de Nariño, Colombia. 2017.

Figure 2. Effective mean dose value (EC-50) found when evaluating the sensitivity of different concentrations of L. origanoides plant extract in P. infestans isolates. Microbiology laboratory of the research group in Genetics and Evolution of Tropical Organisms (GENPAT) of the Universidad de Nariño, Colombia, 2017.

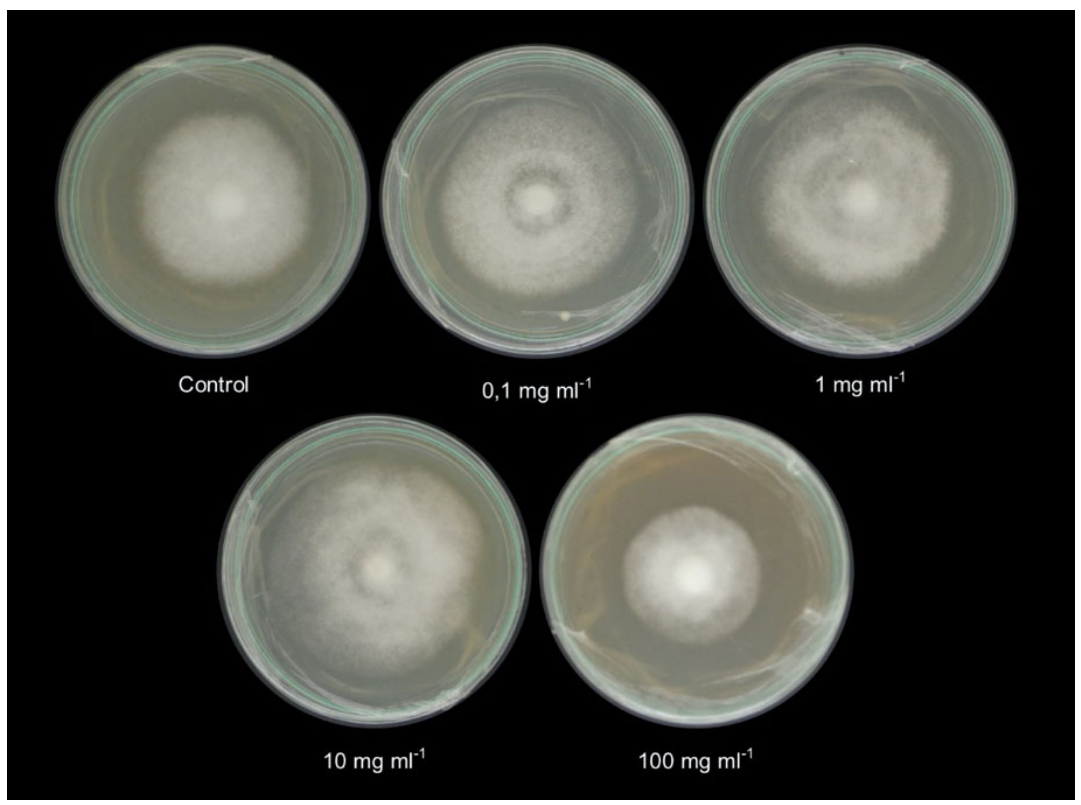

Figura 3. Sensibilidad de Phytophthora infestans (aislamiento STA083) a diferentes concentraciones del extracto acuoso de $O$. vulgare. Laboratorio de microbiología del grupo de investigación en Genética y Evolución de Organismos Tropicales (GENPAT) de la Universidad de Nariño, Colombia. 2017.

Figure 3. Sensitivity of Phytophthora infestans (STA083 isolate) to different concentrations of the aqueous extract of $O$. vulgare. Microbiology laboratory of the research group in Genetics and Evolution of Tropical Organisms (GENPAT) of the Universidad de Nariño, Colombia, 2017. 
comportamiento en las concentraciones de 1 y $10 \mathrm{mg} \mathrm{ml}^{-1}$. En este caso cabe resaltar que el $79 \%$ de los aislamientos presentaron crecimiento en la concentración de $100 \mathrm{mg} \mathrm{ml}^{-1}$ (Cuadro 4), lo que evidenció una baja eficiencia por parte del extracto para el control in vitro de P. infestans en comparación con el comportamiento del patógeno frente al extracto de L. origanoides.

Cuadro 4. Porcentaje de Inhibición de crecimiento radial (PICR), de aislamientos de Phytophthora infestans después de diez días expuesto a extracto acuoso de $O$. vulgare a diferentes concentraciones. Laboratorio de microbiología del grupo de investigación en Genética y Evolución de Organismos Tropicales (GENPAT) de la Universidad de Nariño, Colombia. 2017.

Table 4. Inhibition percentage on radial growth (IPRG), of Phytophthora infestans isolates after ten days exposed to aqueous extract of $O$. vulgare at different concentrations. Microbiology laboratory of the research group in Genetics and Evolution of Tropical Organisms (GENPAT) of the Universidad de Nariño, Colombia, 2017.

\begin{tabular}{|c|c|c|c|c|}
\hline \multirow[b]{2}{*}{ Aislamiento } & \multicolumn{4}{|c|}{ Concentración (mg ml-1) } \\
\hline & 0,1 & 1 & 10 & 100 \\
\hline SPP031 & $7,09 \pm 1,8$ & $24,4 \pm 2,1$ & $29,7 \pm 1,7$ & $56,4 \pm 2,3$ \\
\hline SPT056 & $16,1 \pm 1,6$ & $40,3 \pm 2,3$ & $41,4 \pm 1,4$ & $100 \pm 0$ \\
\hline STA078 & $22,5 \pm 1,5$ & $31,7 \pm 2,4$ & $14,9 \pm 2,2$ & $72 \pm 1,9$ \\
\hline STA083 & $16 \pm 1,1$ & $52,4 \pm 2,9$ & $47,3 \pm 2,8$ & $49 \pm 1,6$ \\
\hline STA084 & $8,7 \pm 0,6$ & $5,9 \pm 0,8$ & $7,7 \pm 0,7$ & $73,4 \pm 0,2$ \\
\hline STA157 & $-0,7 \pm 0,7$ & $9,8 \pm 1,3$ & $45,8 \pm 0,8$ & $54,6 \pm 0,1$ \\
\hline STG075 & $34,3 \pm 0,7$ & $91 \pm 1$ & $90,3 \pm 1,3$ & $82,3 \pm 1,2$ \\
\hline STG100 & $-23,2 \pm 2,6$ & $-4,1 \pm 1,2$ & $12 \pm 2,6$ & $100 \pm 0$ \\
\hline STI003 & $24,4 \pm 0,4$ & $31,2 \pm 1,1$ & $35 \pm 0,4$ & $57,9 \pm 1,3$ \\
\hline STI006 & $-18,9 \pm 1,5$ & $14,4 \pm 3$ & $20 \pm 1,3$ & $43,4 \pm 1,4$ \\
\hline STI155 & $31,4 \pm 0,5$ & $21,5 \pm 1,9$ & $36,2 \pm 0,9$ & $87 \pm 0,2$ \\
\hline STI168 & $13,4 \pm 2,3$ & $51,9 \pm 2,5$ & $58,6 \pm 1$ & $97,5 \pm 1,3$ \\
\hline STP039 & $2,4 \pm 1,1$ & $-0,4 \pm 1,9$ & $69,1 \pm 0,7$ & $97,8 \pm 0,4$ \\
\hline STP041 & $19,7 \pm 0,7$ & $33 \pm 0,5$ & $41,2 \pm 1,6$ & $100 \pm 0$ \\
\hline STP046 & $1,9 \pm 0,2$ & $15,9 \pm 1,7$ & $28,8 \pm 2,5$ & $70,3 \pm 0,6$ \\
\hline STP062 & $7,1 \pm 2,8$ & $21,2 \pm 1,5$ & $26,9 \pm 2,1$ & $82,3 \pm 1,8$ \\
\hline STP067 & $4,4 \pm 2$ & $21,9 \pm 2,5$ & $32,7 \pm 1,9$ & $66,5 \pm 0,8$ \\
\hline STP115 & $3,6 \pm 0,8$ & $-0,03 \pm 1,3$ & $8,2 \pm 2,5$ & $80 \pm 0,5$ \\
\hline STT162 & $7,4 \pm 1,1$ & $4,6 \pm 3,2$ & $54,4 \pm 3,5$ & $100 \pm 0$ \\
\hline
\end{tabular}

* Los datos corresponden a la media de nueve réplicas $(n=9)$; \pm corresponde a la desviación estándar / The data corresponds to the mean of nine replications $(\mathrm{n}=9) ; \pm$ corresponds to the standard deviation.

El análisis de varianza permitió determinar que existieron diferencias altamente significativas $(\mathrm{p}=0,000, \mathrm{gl}=75$, $\alpha=0,05)$ en los porcentajes de inhibición entre las concentraciones de $0,1,1,10$ y la concentración de $100 \mathrm{mg} \mathrm{ml}^{-1}$, formando dos grupos diferentes.

En cuanto a las tasas de esporulación se observó que el $32 \%$ de los aislamientos tuvieron la capacidad de producir esporangios en la concentración de $100 \mathrm{mg} \mathrm{ml}^{-1}$. El análisis estadístico de Kruskal-Walis mostró diferencias estadísticamente representativas $(\mathrm{p} \leq 0,05)$, las cuales se presentaron entre la concentración más alta evaluada (100 $\mathrm{mg} \mathrm{ml}^{-1}$ ) y las demás concentraciones $\left(0,0,1,1\right.$ y $\left.10 \mathrm{mg} \mathrm{ml}^{-1}\right)$ (Cuadro 5). Adicionalmente, en todos los casos se 
Cuadro 5. Prueba Kruskal-Wallis (Bonferroni corrected) para tasa de esporulación entre concentraciones del extracto acuoso de $O$. vulgare. Laboratorio de microbiología del grupo de investigación en Genética y Evolución de Organismos Tropicales (GENPAT) de la Universidad de Nariño, Colombia. 2017.

Table 5. Kruskal-Wallis test (Bonferroni corrected) for sporulation rate between concentrations of the aqueous extract of $O$. vulgare. Microbiology laboratory of the research group in Genetics and Evolution of Tropical Organisms (GENPAT) of the Universidad de Nariño, Colombia, 2017.

\begin{tabular}{cccccc}
\hline Concentración $\left(\mathbf{m g ~ m}^{-1}\right)$ & Control & $\mathbf{0 , 1}$ & $\mathbf{1}$ & $\mathbf{1 0}$ & $\mathbf{1 0 0}$ \\
\hline Control & 1 & & & & \\
$\mathbf{0 , 1}$ & 1 & 1 & & & \\
$\mathbf{1}$ & 1 & 1 & 1 & & \\
$\mathbf{1 0}$ & 1 & 1 & 1 & 1 & \\
$\mathbf{1 0 0}$ & 0,0001036 & 0,0005078 & 0,003361 & 0,01367 & 1 \\
\hline
\end{tabular}

evidenció una alta producción de esporangios con tasas de esporulación promedio de 11 518, 8583, 9428, 8305 y 2076 esporangios $\mathrm{cm}^{-2}$ para las concentraciones de $0,0,1,1,10$ y $100 \mathrm{mg} \mathrm{ml}^{-1}$, respectivamente.

En cuanto a los valores de la dosis media efectiva (EC-50), el valor promedio de la EC-50 fue de 35,6 mg $\mathrm{ml}^{-1}$, con un valor máximo de 214,8 para el aislamiento STI006 y un valor mínimo de $0,1 \mathrm{mg} \mathrm{ml}^{-1}$ en el caso del aislamiento STG075 (Figura 4). Los altos valores presentados en esta variable se debieron a la capacidad del patógeno de crecer en concentraciones de hasta $100 \mathrm{mg} \mathrm{ml}^{-1}$.

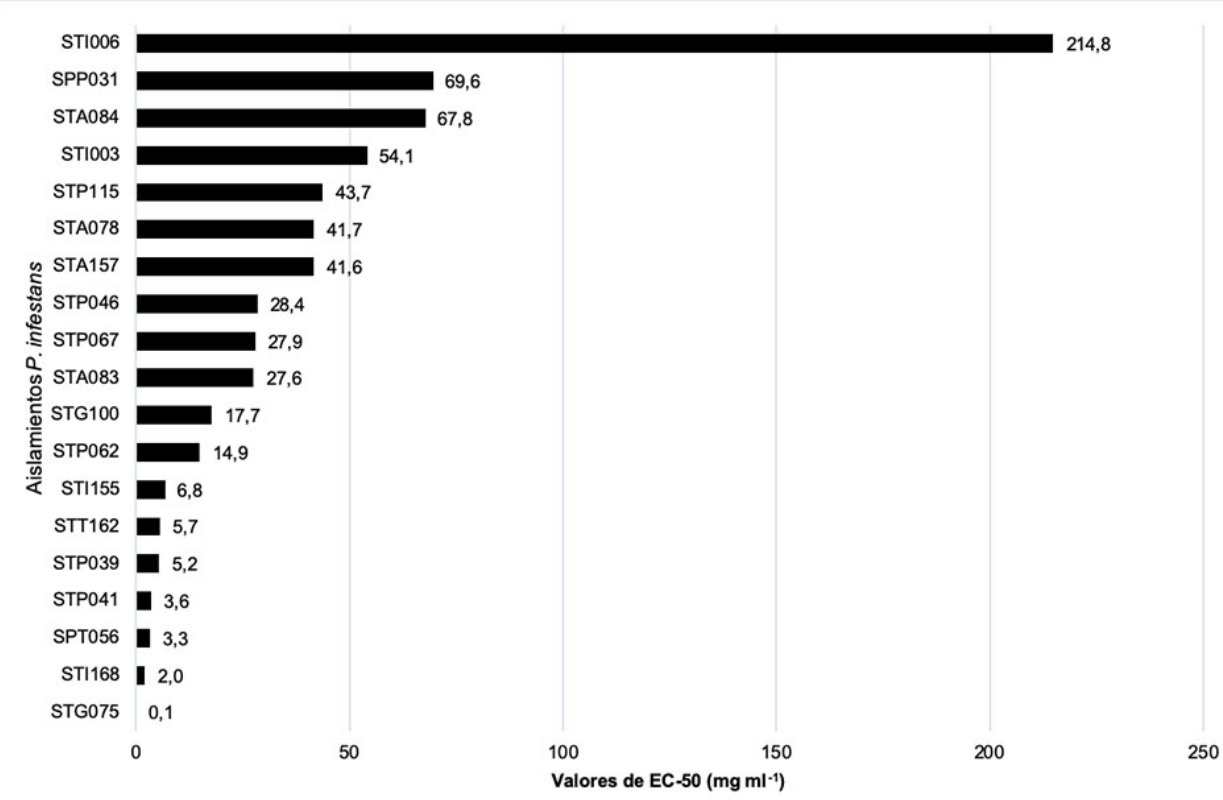

Figura 4. Valor dosis media efectiva (EC-50) encontrados al evaluar la sensibilidad de diferentes concentraciones de extracto vegetal de $O$. vulgare en aislamientos de Phytophthora infestans. Laboratorio de microbiología del grupo de investigación en Genética y Evolución de Organismos Tropicales (GENPAT) de la Universidad de Nariño, Colombia. 2017.

Figure 4. Effective mean dose value (EC-50) found when evaluating the sensitivity of different concentrations of $O$. vulgare plant extract in P. infestans isolates. Microbiology laboratory of the research group in Genetics and Evolution of Tropical Organisms (GENPAT) of the Universidad de Nariño, Colombia, 2017. 


\section{Discusión}

Los resultados obtenidos mostraron que el extracto acuoso de L. origanoides tuvo la capacidad de controlar el crecimiento micelial y la esporulación de $P$. infestans en condiciones in vitro. Esta eficiencia pudo relacionarse con la composición química del extracto obtenido a partir de la biomasa aérea, donde monoterpenos fenólicos como carvacrol y timol son los principales constituyentes (Stashenko et al., 2010; Oliveira et al., 2006; Vicuña et al., 2010), con cantidades relativas del 1,7 y 54,5\% respectivamente, característicos del quimiotipo timol presente en el departamento de Nariño (Betancur-Galvis et al., 2011; Ramírez et al., 2011). Estos compuestos pudieron ser los responsables de que el extracto actuara como inhibidor del patógeno, debido a su actividad biológica (BetancurGalvis et al., 2011; Nostro et al., 2004; Oliveira et al., 2007).

El efecto inhibidor tanto en el crecimiento como en la esporulación por parte de este extracto, pudo estar relacionado con la presencia y concentración de los componentes fenólicos mencionados, los cuales han reportado actividad antifúngica (Arango et al., 2015; Arámbula et al., 2019; Wang et al., 2019). En cuanto a los valores de EC-50, los resultados contrastaron con lo reportado por Arango et al. (2015), quienes encontraron valores de EC-50 de $20,53 \mu \mathrm{g} \mathrm{ml}^{-1}$ cuando el medio de cultivo se suplementó con aceite esencial de orégano silvestre a concentraciones de 10,50,100,150, 200 y $250 \mathrm{gg} \mathrm{ml}^{-1}$, estas diferencias se debieron probablemente a que la composición y la concentración de los compuestos fenólicos fue variable y pudo afectarse por factores ambientales como características del suelo o condiciones climáticas (Arango et al., 2011), así como también con el método de obtención del extracto (Bedoya et al., 2012).

En el caso de $O$. vulgare, la capacidad inhibitoria en el crecimiento y en la esporulación de P. infestans pudo estar asociada con una moderada actividad fitotóxica, como lo reportaron Mancini et al. (2014). Dicha actividad biológica a menudo se atribuyó también a la presencia de los compuestos fenólicos bioactivos timol y carvacrol (Arámbula et al., 2019; Esen et al., 2007; García-Beltrán \& Esteban, 2016; Mancini et al., 2014; Soylu et al., 2006). Estos compuestos se caracterizan por tener una actividad antimicrobiana más eficiente en comparación con otros metabolitos secundarios no fenólicos (Ultee et al., 2002; Busquet et al., 2005).

Los dos compuestos fenólicos, timol y carvacrol, se encuentran presentes en los dos extractos evaluados, y los resultados evidenciaron que el extracto obtenido a partir de L. origanoides fue más eficiente que $O$. vulgare en el control in vitro de $P$. infestans, esto se debió probablemente a la posición del grupo hidroxilo, la cual tiene un efecto importante en la actividad antifúngica (Kim et al., 2008). De la misma manera, se pudo relacionar con la composición química, la cual depende de la procedencia del material vegetal y el método de obtención del extracto (Ramírez-González et al., 2011).

Existió actividad antifúngica por parte de los extractos acuosos evaluados frente a $P$. infestans en condiciones in vitro, por esta razón se consideraron como potenciales biofungicidas para el control de la enfermedad conocida como tizón tardío de la papa. Adicionalmente, es importante resaltar que la especie L. origanoides es una planta de fácil establecimiento que se encuentra en estado silvestre en el norte de Sur América y América Central (Stashenko \& Martínez, 2019), la cual presenta una alta actividad biológica en varios campos de estudio y que se encuentra distribuida en varios departamentos de Colombia, sin embargo, este tipo de plantas requiere que se desarrollen planes para su producción masiva y estudios que permitan evaluar el comportamiento a nivel in planta y en campo para determinar su eficacia, fitotoxicidad y residualidad, con el fin de proponer productos adecuados para una producción limpia.

\section{Conclusiones}

En condiciones in vitro los extractos acuosos de L. origanoides y $O$. vulgare presentaron un efecto antifúngico sobre el oomycete $P$. infestans. 
La reducción del crecimiento radial y la esporulación en los aislamientos de P.infestans se observó a partir de la concentración de $10 \mathrm{mg} \mathrm{ml}^{-1}$ en los dos extractos acuosos, con una inhibición completa cuando se empleó el extracto de L. origanoides a una concentración de $100 \mathrm{mg} \mathrm{ml}^{-1}$.

La dosis media efectiva promedio del extracto acuoso de L. origanoides que permitió reducir el crecimiento del patógeno fue de $5,66 \mathrm{mg} \mathrm{ml}^{-1}$.

\section{Referencias}

Ahmad, A., Khan, A., Akhtar, F., Yousuf, S., Xess, I., Khan, L. A., \& Manzoor, N. (2011). Fungicidal activity of thymol and carvacrol by disrupting ergosterol biosynthesis and membrane integrity against Candida. European journal of clinical microbiology \& infectious diseases, 30(1), 41-50. https://doi.org/10.1007/s10096-010-1050-8

Arámbula, C. I., Diaz, C. E., \& Garcia, M. I. (2019). Performance, chemical composition and antibacterial activity of the essential oil of Ruta chalepensis and Origanum vulgare. Journal of Physics: Conference Series, 1386(1), 012059. https://doi.org/10.1088/1742-6596/1386/1/012059

Arango, O., Hurtado, A. M., Pantoja, D., \& Santacruz, L. (2015). Actividad inhibitoria del aceite esencial de Lippia origanoides H.B.K sobre el crecimiento de Phytophthora infestans. Acta Agronómica, 64(2), 116-124. https://doi.org/10.15446/ acag.v64n2.42964.

Arango, O., Hurtado-Benavides, A., \& Toro, I. (2011). Efecto del origen, la época de recolección y la edad de las hojas en el rendimiento y el contenido de timol de aceites esenciales de Lippia origanoides HBK. Acta Agronómica, 61(3), $207-213$.

Bedoya, O. A., Bolaños, F., Villota, O., Hurtado, A., \& Toro, I. (2012). Optimización del rendimiento y contenido de timol de aceite esencial de orégano silvestre obtenido por arrastre con vapor. Biotecnología en el Sector Agropecuario y Agroindustrial, 10(2), 217-226.

Betancur-Galvis, L., Zapata, B., Baena, A., Bueno, J., Ruíz-Nova, C. A., Stashenko, E., \& Mesa-Arango, A. C. (2011). Antifungal, cytotoxic and chemical analyses of essential oils of Lippia origanoides HBK grown in Colombia. Revista de la Universidad Industrial de Santander. Salud, 43(2), 141-148.

Busquet, M., Calsamiglia, S., Ferret, A., \& Kamel, C. (2005). Screening for effects of plant extracts and active compounds of plants on dairy cattle rumen microbial fermentation in a continuous culture system. Animal Feed Science and Technology, 123-124(2), 597-613. https://doi.org/10.1016/j.anifeedsci.2005.03.008

Dakole, C., Nguefack, J., Dongmo, J., Galani, H., Azah, R., Somda, I., \& Amvam, H. (2016). Antifungal potential of essential oils, aqueous and ethanol extracts of thirteen plants against Fusarium oxysporum f. sp Lycopersici and Phytophtora infestans (Mont.) de Bary as major tomato pathogens in Cameroon. International Journal of Current Science, 19(2), 128-145.

Debjani, C., Prerna, D., Seweta, S., Soumen, S., \& Susamoy, K. (2018). Role of botanical plant extracts to control plant pathogens-A review. Indian Journal of Agricultural Research, 52(4), 341-346. https://doi.org/10.18805/IJARe.A-5005

Esen, G., Azaz, A. D., Kurkcuoglu, M., Baser, K. H. C., \& Tinmaz, A. (2007). Essential oil and antimicrobial activity of wild and cultivated Origanum vulgare L. subsp. hirtum (Link) letswaart from the Marmara region, Turkey. Flavour and Fragrance Journal, 22(5), 371-376. https://doi.org/10.1002/ffj.1808

García, H. G., Marín, M., Jaramillo, S., \& Cotes, J. M. (2008). Sensibilidad de aislamientos colombianos de Phytophthora infestans a cuatro fungicidas sistémicos. Agronomía Colombiana, 26(1), 47-57. 
García-Beltrán, J. M., \& Esteban, M. A. (2016). Properties and applications of plants of Origanum sp. Genus. SM Journal of Biology, 2(1), 1006-1015.

García-Camarillo, E. A., Quezada-Viay, M. Y., Moreno-Lara, J., Sánchez-Hernández, G., Moreno-Martínez, E., \& Pérez-Reyes, M. C. J. (2006). Actividad antifúngica de aceites esenciales de canela (Cinnamomum zeylanicum Blume) y orégano (Origanum vulgare L.) y su efecto sobre la producción de aflatoxinas en nuez pecanera [Carya illinoensis (FA Wangenh) K. Koch]. Revista Mexicana de Fitopatología, 24(1), 8-12.

Garrett, K. A., Nelson, R. J., Mundt, C. C., Chacon, G., Jaramillo, R. E., \& Forbes, G. A. (2001). The effects of host diversity and other management components on epidemics of potato late blight in the humid highland tropics. Phytopathology, 91(10), 993-1000. https://doi.org/10.1094/PHYTO.2001.91.10.993

International Panel of Experts on Sustainable Food Systems / Food and Agriculture Organization. (2010). Biopreparados para el manejo sostenible de plagas y enfermedades en la agricultura urbana y periurbana. http://www.fao.org/3/a-as $435 \mathrm{~s} . \mathrm{pdf}$

Kim, J., Lee, Y. S., Lee, S. G., Shin, S. C., \& Park, I. K. (2008). Fumigant antifungal activity of plant essential oils and components from West Indian bay (Pimenta racemosa) and thyme (Thymus vulgaris) oils against two phytopathogenic fungi. Flavour and Fragrance Journal, 23(4), 272-277. https://doi.org/10.1002/ffj.1882

Mancini, E., Camele, I., Elshafie, H. S., De Martino, L., Pellegrino, C., Grulova, D., \& De Feo, V. (2014). Chemical composition and biological activity of the essential oil of Origanum vulgare ssp. hirtum from different areas in the Southern Apennines (Italy). Chemistry \& Biodiversity, 11(4), 639-651. https://doi.org/10.1002/cbdv.201300326

Mohsan, M., Ali, S., Shahbaz, M. U., Saeed, S., \& Burhan, M. (2017). In vitro efficacy of different growth media and crude plant extracts against mycelia growth of Phytophthora capsici. Journal of Applied Biology \& Biotechnology, 5(04), 043-047. https://doi.org/10.7324/JABB.2017.50407

Nagar, D., Biswas, S.K., Singh, M., \& Singh, J. (2017). Evaluation of in-vitro Efficacy of Different Plant Extracts against Phytophthora infestans (Mont.) de Bary. International Journal of Current Microbiology and Applied Sciences, 6(6), 412-417. https://doi.org/10.20546/ijcmas.2017.606.048

Nostro, A., Blanco, A. R., Cannatelli, M. A., Enea, V., Flamini, G., Morelli, I., Roccaro, A. S., \& Alonzo, V. (2004). Susceptibility of methicillin-resistant staphylococci to oregano essential oil, carvacrol and thymol. FEMS Microbiology Letters, 230(2), 191-195. https://doi.org/10.1016/S0378-1097(03)00890-5

Numpaque, M. A., Oviedo, L. A., Gil, J. H., García, C. M., \& Durango, D. L. (2011). Thymol and carvacrol: biotransformation and antifungal activity against the plant pathogenic fungi Colletotrichum acutatum and Botryodiplodia theobromae. Tropical Plant Pathology, 36(1), 3-13. http://dx.doi.org/10.1590/S1982-56762011000100001

Ochoa-Fuentes, Y. M., Cerna-Chávez, E., Landeros-Flores, J., Hernández-Camacho, S., \& Delgado-Ortiz, J. C. (2012). Evaluation in vitro of the anti-fungal activity of four methanol plant extracts for the control of three species of Fusarium spp. Phyton, 81, 69-73.

Oliveira, D. R., Leitao, G. G., Bizzo, H. R., Alviano, D. S., Alviano, C. S., \& Leitão, S. G. (2007). Chemical and antimicrobial analyses of essential oil of Lippia origanoides HBK. Food Chemistry, 101(1), 236-240. https://doi.org/10.1016/j. foodchem.2006.01.022

Oliveira, D. R., Leitão, G. G., Santos, S. S., Bizzo, H. R., Lopes, D., Alviano, C. S., Alviano, D. S., \& Leitão, S. G. (2006). Ethnopharmacological study of two Lippia species from Oriximiná, Brazil. Journal of Ethnopharmacology, 108(1), 103-108. https://doi.org/10.1016/j.jep.2006.04.018 
Ramírez, L. S., Isaza, J. H., Veloza, L. Á., Stashenko, E., \& Marín, D. (2011). Actividad antibacteriana de aceites esenciales de Lippia origanoides de diferentes orígenes de Colombia. Ciencia, 17(4), 313-321.

Ramírez-González, S. I., López-Báez, O., de Jesús Guzmán-Hernández, T., Munguía-Ulloa, S., \& Espinosa-Zaragoza, S. (2011). Actividad antifúngica in vitro de extractos de Origanum vulgare L., Tradescantia spathacea Swartz y Zingiber officinale Roscoe sobre Moniliophthora roreri (Cif \& Par) Evans et ál. Revista Tecnología en Marcha, 24(2), 3-17.

Red de Información y Comunicación del Sector Agropecuario de Colombia. (2018). Área, producción y rendimiento nacional por cultivo. https://www.agronet.gov.co/estadistica/Paginas/home.aspx?cod=1

Rivera, H. F., Martínez, E. P., Osorio, J. A., \& Martínez, E. (2010). Respuesta de biosurfactantes producidos por Pseudomonas fluorescens para el control de la gota de la papa Phytophthora infestans (Mont) de Bary, bajo condiciones controlada. Ciencia \& Tecnología Agropecuaria, 11(1),21-30. https://doi.org/10.21930/rcta.vol11_num1_art:191

Santa, J. D., Berdugo-Cely, J., Cely-Pardo, L., Soto-Suárez, M., Mosquera, T., \& Galeano C. H. (2018). QTL analysis reveals quantitative resistant loci for Phytophthora infestans and Tecia solanivora in tetraploid potato (Solanum tuberosum L.). Plos One, 13(7), e0199716. https://doi.org/10.1371/journal.pone.0199716

Silva, B., Jaramillo, S., \& Marín, M. (2009). Caracterización genética de aislamientos de Phytophthora infestans en las zonas productoras de papa de los departamentos de Antioquia, Boyacá, Cundinamarca y Norte de Santander (Colombia). Actualidades Biológicas, 31(90), 5-20.

Soylu, E. M., Soylu, S., \& Kurt, S. (2006). Antimicrobial activities of the essential oils of various plants against tomato late blight disease agent Phytophthora infestans. Mycopathologia, 161(2), 119-128. https://doi.org/10.1007/s11046-005-0206-z

Stashenko, E. E., Martínez, J. R., Ruíz, C. A., Arias, G., Durán, C., Salgar, W., \& Cala, M. (2010). Lippia origanoides chemotype differentiation based on essential oil GC-MS and principal component analysis. Journal of Separation Science, 33(1), 93-103. https://doi.org/10.1002/jssc.200900452

Stashenko, E. E., \& Martínez, R. J. (2019). Study of essential oils obtained from Tropical plants grown in Colombia. In ElShemy, H. (Ed.), Essential Oils-Oils of Nature. (pp. 403-451). IntechOpen. https://doi.org/10.5772/intechopen.87199

Ultee, A., Bennik, M. H. J., \& Moezelaar, R. (2002). The phenolic hydroxyl group of carvacrol is essential for action against the food-borne pathogen Bacillus cereus. Applied and Environmental Microbiology, 68(4), 1561-1568. https://doi. org/10.1128/AEM.68.4.1561-1568.2002

Vicuña, G. C., Stashenko, E. E., \& Fuentes, J. L. (2010). Chemical composition of the Lippia origanoides essential oils and their antigenotoxicity against bleomycin-induced DNA damage. Fitoterapia, 81(5), 343-349. https://doi.org/10.1016/j. fitote. 2009.10 .008

Wang, K., Jiang, S., Pu, T., Fan, L., Su, F., \& Ye, M. (2019). Antifungal activity of phenolic monoterpenes and structure-related compounds against plant pathogenic fungi. Natural Product Research, 33(10), 1423-1430. https://doi.org/10.1080/1 4786419.2017.1419232

Widmer, T. L., \& Laurent, N. (2006). Plant extracts containing caffeic acid and rosmarinic acid inhibit zoospore germination of Phytophthora spp. pathogenic to Theobroma cacao. European Journal of Plant Pathology, 115(4), Article 377. https:// doi.org/10.1007/s10658-006-9024-5

You, J., Zhang, J., Wu, M., Yang, L., Chen, W., \& Li, G. (2016). Multiple criteria-based screening of Trichoderma isolates for biological control of Botrytis cinerea on tomato. Biological Control, 101, 31-38. https://doi.org/10.1016/j. biocontrol.2016.06.006

162 